\title{
Psychophysiological Analysis (PSPHA): A modular script-based program for analyzing psychophysiological data
}

\author{
ARMAND DE CLERCQ, BRUNO VERSCHUERE, PETRA DE VLIEGER, and GEERT CROMBEZ \\ Ghent University, Ghent, Belgium
}

\begin{abstract}
In psychophysiological research, complex tailor-made and interactive analyses of biosignals (e.g., skin conductance, heart rate, and respiration) are often required. Moreover, a synchronization between experimental stimuli and psychophysiolgical responses is necessary. In this article, we present Psychophysiological Analysis (PSPHA), a modular script-based program for analyzing biosignals in the time domain. The modules can be integrated in a VBScript, and a wizard allows easy adaption of parameters. PSPHA is a free, interactive, and flexible program for analyzing the data of psychophysiological experiments.
\end{abstract}

Psychophysiology covers a broad range of research topics, ranging from stress reactivity to the detection of deception. Often, researchers investigate the effect of an experimental manipulation (e.g., lying vs. answering truthfully) on physiological signals, such as sweating, respiration, brain waves, or cerebral blood flow. A number of software packages exist to analyze psychophysiological data. Some of these packages are for use in clinical settings, in which one is interested mainly in steady state signals. In experimental studies, the immediate change of physiological parameters as a response to an event is of critical importance. Several commercial and noncommercial programs exist that allow synchronization between experimental stimuli and physiological responses. Examples of commercial programs are MacLab/PowerLab (ADInstruments, 2004), software from J\&J Engineering (J\&J Engineering, 2004), BioGraph (Thought Technology, 2004), and PSYLAB (Contact Precision Instruments, 2004). Disadvantages of these programs are the cost and the fact that they are offered along with particular hardware instrumentation. Examples of noncommercial programs are SCORIT (Prokasy, 1974), SCRGAUGE (Boucsein, 1992), software as described by Storm, Fremming, Ødegården, Martinsen, and Mørkrid (2000), SCORES (Lim et al., 1999), and PSAAL (Angrilli, 1995). Most often, these software programs are written for one particular psychophysiological signal and do not allow the analysis of multiple signals and/or their interaction.

Our program for analyzing psychophysiological responses, PSPHA, overcomes these disadvantages: (1) It is

Correspondence concerning this article should be addressed to A. De Clercq, Department of Applied Mathematics and Computer Science, Ghent University, Krijgslaan 281 S9, B-9000 Ghent, Belgium (e-mail: armand.declercq@ugent.be). written in an internationally accepted computer language (Visual Basic 6 [VBS]), (2) it allows synchronization between experimental stimuli and psychophysiological responses, (3) it allows improvement of the signal-to-noise ratio (e.g., through smoothing or finite impulse response [FIR] filtering) and data reduction of multiple signals and their interaction, and (4) it is flexible, interactive, and free to use. At present, PSPHA is user ready for analyzing electrodermal, cardiac, and respiratory data. PSPHA can, however, easily be extended to other measures (e.g., electromyography recordings [EMG]) or the interaction between measures (e.g., respiratory sinus arrhythmia).

PSPHA reads data sets that are created with VPM (Cook, Atkinson, \& Lang, 1987), but because VPM is not a generally used format, reading data sets in a delimited format is preferred. Most other data forms may be converted to such a delimited format. For signal enhancement and data reduction, data are displayed on screen. In PSPHA, all or a set of selected channels can be displayed. The data can be zoomed or panned. Figure 1 shows a typical window. Also, experimental markers, which are used to indicate the occurrence of external events, such as the beginning or ending of a stimulus, the subjects' response, or noise (e.g., coughing), can be displayed or hidden. The program provides an easy-to-handle format for data editing and parameter extraction. Data analyses are performed by selecting an item in the script menu that activates VBSscript subroutines that are created for the particular experiment. PSPHA consists of a limited number of base functions. The most important ones are those for calculating minima and maxima (or alternatively, inflection points) within particular time windows. In many situations, these functions are sufficient to calculate the most widely used physiological parameters, such as SCR amplitude, T-wave, or R-peak in the electrocardiogram 


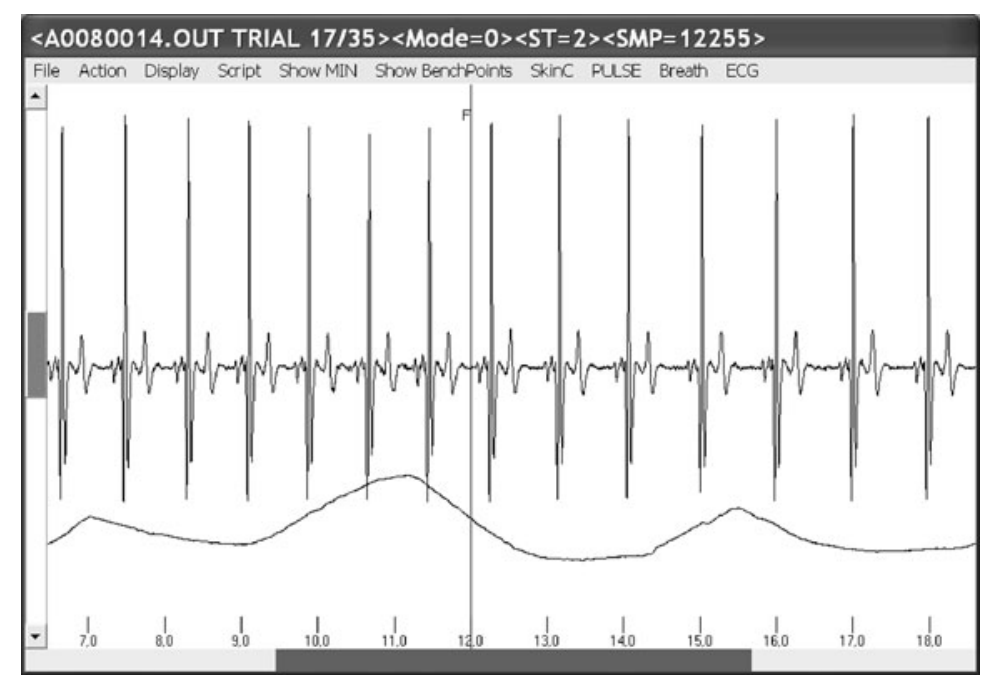

Figure 1. A typical PSPHA window displaying an experimental marker indicating the onset of an event and two psychophysiological signals. The upper trace represents heart rate data (ECG). The lower trace represents respiration data.

(ECG) signal or the depth of breathing (amplitude). Other functions may, however, be easily added and implemented using VBScript. After parameter extraction, the physiological parameters are stored as internal markers (e.g., MIN, MAX, ...) and added to the screen. This strategy allows the experimenter to visually inspect and correct the data and the proposed parameters. Finally, the data and parameter output are converted to any format for processing by statistical packages.

PSPHA is written in Visual Basic and VBScript. We have created a number of predefined scripts that probably are applicable in most psychophysiological experiments. An example of a predefined script will be discussed in the User Interface section. The VBScripts can, however, be modified by the user for particular applications. Figure 2 shows that a number of PSPHA parameters can be changed directly without need to reprogram VBScripts.

\section{Object-Based Concept}

PSPHA uses a hierarchical object-oriented approach for storing and analyzing data. Data are read in the program as a collection of trial objects. Each trial object consists of a collection of parameters for storing general information (e.g., trial number, participant number, and SCR amplitude) and a collection of data objects, one for each physiological channel. The data object contains the binary data but also marker objects. Marker objects are of crucial importance for data reduction. Experimental markers indicate the occurrence of an external event. Internal markers refer to calculated items, such as minima and maxima.

The object approach is maintained for data storage by using the XML format. XML (World Wide Web Consortium, 2005) is a standard way of defining formats for and storing semistructured data. Storing data in XML format has the advantage that their structure may be examined by using a Web browser such as Microsoft Internet Explorer. Because all the data are presented in textual form, the file length is larger than when data are stored in binary form. The size is approximately 2.5 times larger, but this is no problem for current PCs.

\section{Markers}

Data analysis is based on the markers. Markers are objects with a time attribute. Experimental markers represent time properties of external events, such as the beginning of a trial, the end of a trial, or the beginning of an external event, such as a stimulus. Internal markers are often specific for a particular data channel, such as the exact time of a minimum, maximum, or inflection point in the electrodermal channel. A minimum and maximum is defined as the point in the curve where the first derivative equals zero. An inflection is defined as the point where the second derivative equals zero.

An example of the XML representation of the amplitude (maximum) for skin conductance is $<\mathrm{X} 5 \mathrm{~A} 0=" \mathrm{M} "$ $\mathrm{A} 1=$ "MAX" A2 ="V=17650;E $=+348.68 ; \mathrm{S}=\mathrm{OK} ; \mathrm{C}=1$ $6711935 " \mathrm{~A} 3=" \mathrm{U} " />$.

$\mathrm{A} 0=$ " $\mathrm{M}$ " indicates that this is a marker; the value of $\mathrm{A} 1=$ "MAX" indicates a maximum; A2 contains a text string that represents additional information; $\mathrm{V}$ is the time of the maximum; $\mathrm{E}$ is the amplitude of the maximum; $\mathrm{S}=$ "OK" indicates that this is a valid marker; $\mathrm{C}$ denotes the color for the representation on screen; and the value "U" of A3 indicates that the marker was generated by the user self. Internal markers can be removed and recalculated, whereas experimental markers cannot. Notice that additional information is represented as one single XML attribute, A2. An advantage of this option is that additional information can always be added without altering the basic structure of the data. 


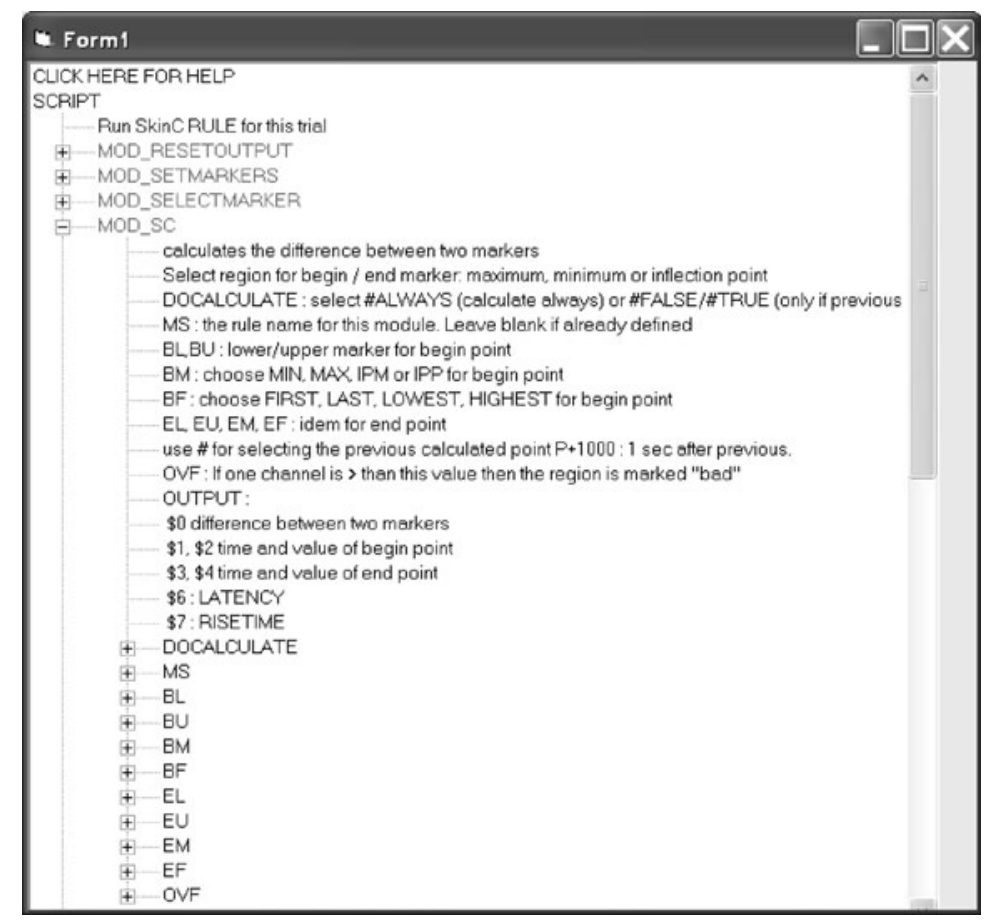

Figure 2. Window for modifying module parameters.

\section{Parameters}

Additional data may also be stored as parameter objects. The following is an illustration of this. An experimenter is interested in a change in heart rate in response to the presentation of a set of pictures (e.g., emotional vs. neutral). Internal markers (maxima) are used to detect the R-peaks in the ECG signal. Next, the distance between these maxima are calculated in order to obtain the interbeat intervals (IBIs). A comparison of the IBIs immediately after slide presentation with those before the presentation of the stimulus (baseline) allows the experimenter to calculate the second-to-second change in heart rate. The final parameter may, for example, be the largest decline in heart rate in the 10 -sec poststimulus period.

The XML structure of parameter objects is the same as that of marker objects. A difference is that marker objects are always defined for one particular data channel, whereas parameter objects may be defined both globally (for the entire experiment) and locally (for one particular trial).

\section{Parameter Extraction of Psychophysiological Data}

Skin conductance response: Calculation after smoothing. The amplitude of the skin conductance response to a particular stimulus is calculated by using the time and the value of a maximum, which is compared with the time and value of a preceding minimum. The default settings are those that are recommended by Dawson, Schell, and Filion (2000): Only minima occuring within $1-5 \mathrm{sec}$ after stimulus onset that are followed by a maximum $1-5 \mathrm{sec}$ later are considered valid. These settings can, however, be changed. At present, PSPHA is used only to calculate the amplitude of the electrodermal response, but the user can easily adopt PSPHA to calculate other parameters (e.g., latency, rise time, or half-recovery time).

Before calculation of the maxima and the minima, the data are smoothed with a second degree polynomial: $\mathrm{Y}=\mathrm{C} 1+\mathrm{C} 2 * \mathrm{X}+\mathrm{C} 3 * \mathrm{X} * \mathrm{X}$. The Marquardt least-square fitting procedure is used. We translated available routines for Marquardt least-square fitting that were written in C (Press, Flannery, Teukolsky, \& Vetterling, 1993), into VBS. The number of fitting points can easily be changed by the user. Traditionally, skin conductance is smoothed by using FIR filters (Cook \& Miller, 1992). FIR filters can be used in PSPHA, but we prefer the Marquardt algorithm. This algorithm calculates the first derivative in each point and is used to identify inflection points. Inflection points may be helpful for detecting changes in skin conductance whenever a maximum is not easily detected. Another advantage is the possibility of fitting more sophisticated functions (see Lim et al., 1997). However, FIR filters are easy to implement. We use FIR filters, for example, for filtering EMG signals.

After smoothing, maxima and minima are calculated by identifying the highest or lowest point in an indicated region. In the absence of minima or maxima, negative or positive inflection points are used. When neither a maximum nor a positive inflection point can be found after a minimum or a negative inflection point, the trial will be scored as a 0-response, indicating that the stimulus did not elicit a response.

Heart rate. In PSPHA, heart rate data are analyzed in the time domain. Frequency analysis (e.g., fast Fourier 
transform) is not yet implemented. Heart rate data are calculated without smoothing in PSPHA, because the standard is to use a hardware band-pass filter (e.g., 8-40 Hz) in the amplifier. First, PSPHA detects the highest maxima (= the R-peak) in the ECG signal. Only maxima that are followed by a minimum are considered valid. PSPHA calculates the distances between the maxima, and the resulting IBIs are converted to heart rate in beats per minute (bpm) per real-time epoch. The standard procedure of Graham (1978) is followed.

One of the potential problems with heart rate data is the presence of artifacts: R-peaks can be missing, or other maxima (e.g., the P- or T-peak) can be misinterpreted as R-waves. A number of procedures are available for artifact detection and signaling the user of their potential presence. The criteria used here conform with those in Stekelenburg and Van Boxtel (2001). If an IBI is larger than 1,500 msec (40 bpm) or the interval is greater than $130 \%$ of the mean of the surrounding IBIs, PSPHA signals the user that there may be a missing maximum. The experimenter may choose, then, between several options to deal with the problem of a missing maximum: (1) The experimenter may mark the data section as invalid; (2) he/she may add a maximum between the surrounding maxima; or (3) he/she may decide to accept the data as valid. In our experience, missing maxima occur exceptionally because maxima that were not followed by the obligatory minimum are deleted. In that case, the data can be reprocessed for that particular trial without the artifact detection procedure. If an IBI is less than $400 \mathrm{msec}(150 \mathrm{bpm})$ or the interval is shorter than $70 \%$ of the mean of the surrounding IBIs, PSPHA signals the user that a maximum may have been erroneously detected. Again, the user has several options for handling an erroneous maximum: (1) He/she may mark the data section as invalid; (2) he/she may delete the maximum; or (3) he/she may accept the maximum as valid.
Respiration. PSPHA may also be used for an interactive analysis of respiration. It calculates respiratory amplitude (depth of breathing) and respiratory period (duration of breathing). Figure 3 shows a computer-generated breath cycle, consisting of inspiration (indicated by a decline), expiration, and an expiratory pause. Some respiratory parameters are displayed. Respiratory amplitude is calculated by comparing the maximum of the breath cycle with its preceding minimum. Respiratory period is calculated by comparing the timing of the minimum with its preceding minimum. On the basis of these parameters, a number of other parameters, such as inspiration period, expiration period, and inspiratory flow, can easily be calculated and are already implemented in PSPHA.

Figure 3 also illustrates the fact that there is often not a well-defined single maximum in the expiratory phase. Simply calculating the maximum would remove valuable information about the start and the end of this maximum. In order to preserve this information, PSPHA fits the prolonged maximum with a combination of two half-Gauss curves: one half for the left side and another one for the right side of the curve. The top of both half-Gauss functions, which need to be the same, are connected with a straight horizontal line. Figure 4 shows an expiratory pause that is fitted with two half-Gauss functions. In some cases, the fit is not as expected. The experimenter may, then, manually change the left and the right wings of the region before analyzing the data.

\section{User Interface}

Reading text-delimited data is preferred. The information for reading the text files has to be created with PSPHADELIM. This program adds an information line to a list from which the user can select one for reading the data file. In this step, the original data are converted to the XML form. On the next occasion, the XML file can

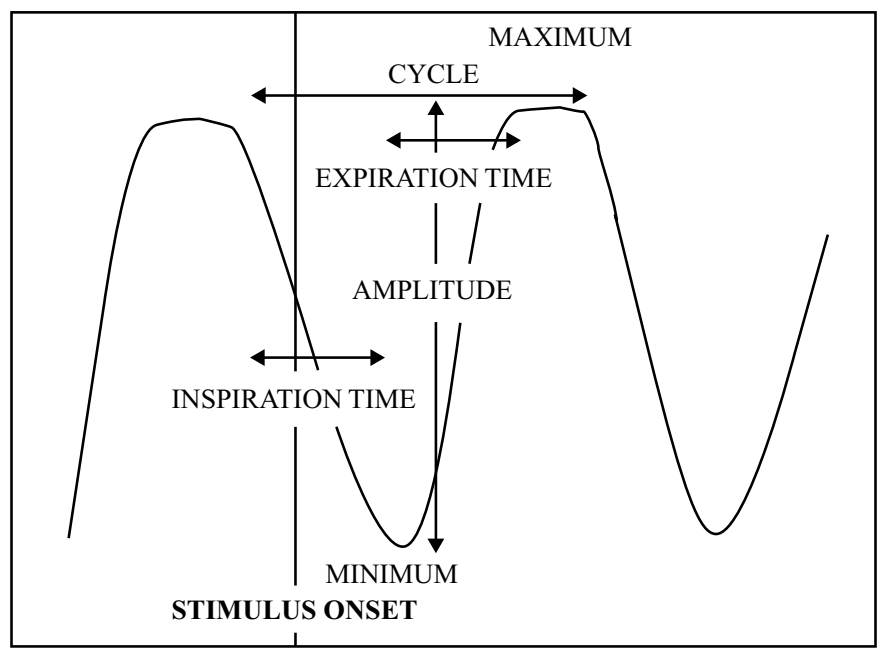

Figure 3. A computer-generated example of respiration data. The following respiratory parameters are displayed: the minimum, maximum, and amplitude of a respiratory cycle and the inspiration and expiration time of a respiratory cycle. 


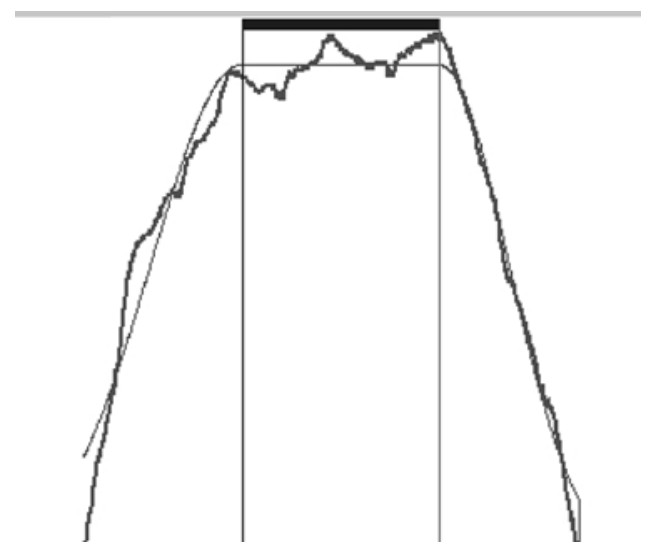

Figure 4. Expiratory pause in the respiration curve with halfGauss fit.

be used immediately. In PSPHA, all or selected channels can be displayed, the data can be zoomed and panned, and markers can be shown or hidden. Data analyses are performed by selecting an item in the script menu that activates VBScript subroutines that are created for the experiment. An example script for analyzing SCR data is given in Appendix A. The link of the data with one particular script is done in PSPHADELIM during a format definition phase.

Base modules. There are more than 10 script modules available for analyzing psychophysiological experiments. The script modules given in Appendices B and C are exemplary. They make use of subroutines, which are described in detail in the PSPHA help file (allserv.Ugent. be/ adeclerc/PSPHA/HELP). Using these subroutines, all parameters in PSPHA can be called and defined. All markers can be read, and new markers can be generated. Base subroutines for calculating maxima, minima, inflection points, and regions are available, and special subroutines for calculating heart rate parameters already exist. As an example, we added the two modules in Appendices B and $\mathrm{C}$. We removed comments and lines that are functional only when the modules are used in other situations. Comments are essential parts of the script because they appear in the form of messages for the end user, describing the functionality of each module (see Figure 2).

Internationalization. In some European countries, the decimal separator is the comma and not the point. VBScript uses the local country sign when converting numbers to string, but because all data are stored as text in the XML file, numbers are stored with the decimal point. In a VBScript file, parameters containing real data should be accessed with the "PD" name, and not with "P." "PD" converts the textual form (comma or point) in a double number. This number can then be used in the VBScript file without conversion problems. PSPHA will always convert the comma in point again, in the case in which the number is written back.
PSPHADELIM/PSPHAIMP. Since there is no standard for capturing and storing psychophysiological data, it is almost impossible to know the structure of the user's data. Therefore, data must be presented to PSPHA in an ASCII text form. A special program, PSPHADELIM, was created for defining the conversion of those data into the internationally used XML form. Data for different channels can be presented into different files or may be intermixed. In a number of consecutive screens, PSPHADELIM asks for all the parameters that are necessary for reading the data, such as the number of data points in each channel, the number of points on each line, and so forth. The information is stored in a separate XML file. After this stage, data can be read directly in PSPHA by selecting this name when the file is opened for reading. A separate file can be linked together with the data that contains markers for each trial generated in the experiment.

\section{Discussion}

PSPHA offers a freeware alternative for dedicated commercial and noncommercial software for analyzing psychophysiological data. Because experimenters want specific parameters from their data, it is almost impossible to accomplish this in one standard program. Because PSPHA has a modular structure, where relative simple base modules can be integrated together in simple VBScript files, PSPHA tries to fulfill most needs. Furthermore, the whole program is written in an easy-to-learn language, Visual Basic.

\section{Availability}

The software described in this article is available for nonprofit use under the Gnu General Public License (GPL). The terms of the GPL open source license are described at the Web site www.opensource.org/. The complete program, including source, a ready to install binary version, and example data, can be downloaded from allserv.ugent.be/ adeclerc/PSPHA.

\section{REFERENCES}

ADInstruments, Inc. (2004). Powerlab information [Online]. Available at www.adinstruments.com/products/.

ANGRILli, A. (1995). PSAAL: A LabVIEW 3 program for data acquisition and analysis in psychophysiological experiments. Behavior Research Methods, Instruments, \& Computers, 27, 367-374.

BoUCSEIN, W. (1992). SCRGAUGE - a computer program for the detection and quantification of SCR's. In W. Boucsein (Ed.), Electrodermal activity. New York: Plenum.

Contact Precision Instruments Inc. Psylab information [Online]. Available at www.psylab.com/.

Cook, E. W., III, AtKinson, L. S., \& LAnG, K. G. (1987). Stimulus control and data acquisition for IBM PC's and compatibles. Psychophysiology, 24, 726-727.

Cook, E. W., III, \& Miller, G. A. (1992). Digital filtering: Background and tutorial for psychophysiologists. Psychophysiology, 29, 350-367.

Dawson, M. E., Schell, A. M., \& Filion, D. L. (2000). The electrodermal system. In J. T. Cacioppo, L. G. Tassinary, \& G. B. Berntson 
(Eds.), Handbook of psychophysiology (pp. 200-223). Cambridge: Cambridge University Press.

Graham, F. K. (1978). Constraints on measuring heart rate and period sequentially through real and cardiac time. Psychophysiology, 15, 492-495.

J\&J ENGINEERING INC. (2004). Windows software information [Online]. Available at www.jjengineering.com/WinSoftware.htm.

Lim, C. L., Gordon, E., Rennie, C., Wright, J. J., Bahramali, H., Li, W. M., ET AL. (1999). Dynamics of SCR, EEG, and ERP activity in an oddball paradigm with short interstimulus intervals. Psychophysiology, 36, 543-551.

Lim, C. L., Rennie, C., Barry R. J., Bahramali, H., Lazzaro, I., MANOR, B., \& GoRdon, E. (1997). Decomposing skin conductance into tonic and phasic components. International Journal of Psychophysiology, 25, 97-109.

Press, W. H., Flannery, B. P., Teukolsky, S. A., \& Vetterling, W. T.
(1993). Numerical recipes in C: The art of scientific computing. Cambridge: Cambridge University Press.

PROKASY, W. F. (1974). SCORIT: A computer subroutine for scoring electrodermal responses. Behavior Research Methods \& Instrumentation, 6, 49-52.

Stekelenburg, J. J., \& Van Boxtel, A. (2001). Inhibition of pericranial muscle activity, respiration, and heart rate enhances auditory sensitivity. Psychophysiology, 38, 629-641.

Storm, H., Fremming, A., Ødegården, S., Martinsen, Ø. G., \& MøRKRID, L. (2000). The development of a software program for analyzing spontaneous and externally elicited skin conductance changes in infants and adults. Clinical Neurophysiology, 111, 1889-1898.

Thought Technology LtD. (2004). BioGraph Infiniti information [Online]. Available at www.thoughttechnology.com/download.htm. World Wide Web Consortium (2005). Extensible Markup Language (XML) [Online]. Available at www.w3.org/XML/.

\section{APPENDIXA}

VBScript as Used in PSPHA for Analysis

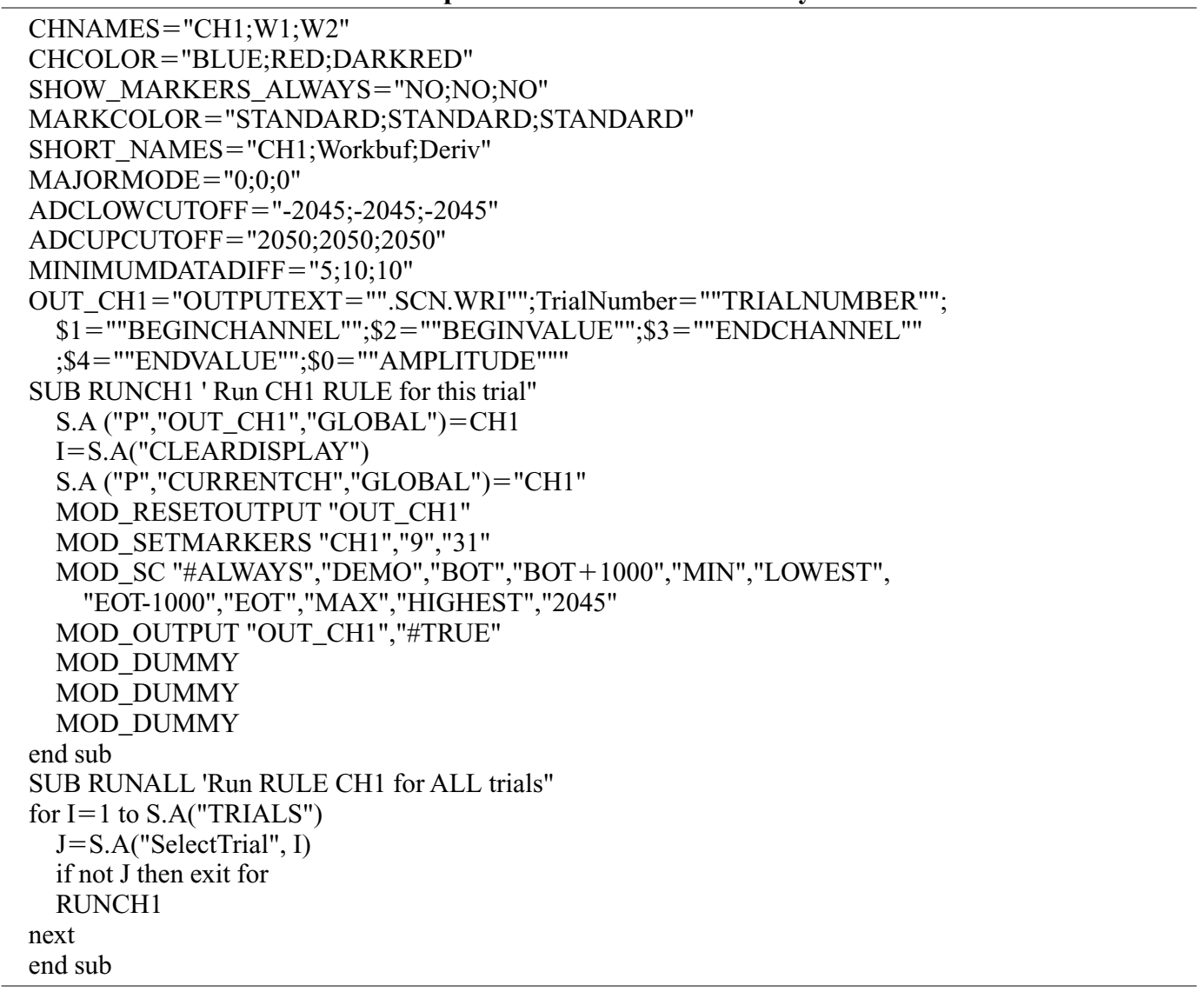




\section{APPENDIX B}

\section{VBScript for MOD_SETMARKERS}

SUB MOD_SETMARKERS (CHANNELNUMBER,SMOOTHPOINTS,MARKERPOINTS)

S.A("P","CURRENTCH","GLOBAL")=CHANNELNUMBER

S.A("DISPLAY","DTONLY")= CHANNELNUMBER

I=S.A("SetSmoothPoints",SMOOTHPOINTS)

I=S.A("SetMarkerPoints",MARKERPOINTS)

END SUB

\begin{tabular}{|c|c|}
\hline \multicolumn{2}{|l|}{$\begin{array}{c}\text { APPENDIX C } \\
\text { VBScript Representing MOD_SC }\end{array}$} \\
\hline SUB MOD_SC (DOCALCULATE,MS,BL,BU,BM,BF,EL,EU,EM,EF,OVF) & 1 \\
\hline ' DOCALCULATE has no meaning if there is just one MOD_SC call & \\
\hline S.A("P","\$0")="9999" & 3 \\
\hline if S.A("CHECKOVERFLOW",BL ,EU,OVF) then & 4 \\
\hline S.A("P","\$\$0")="OVERFLOW" & 5 \\
\hline S.A("F","EXITSTATUS")="\#ERROR" & 6 \\
\hline exit sub & 7 \\
\hline end if & 8 \\
\hline S.A("F","EXITSTATUS")="\#TRUE" & 9 \\
\hline BEGP= S.A("SetMark",BL,BU,BM,BF,"\$1","\$2","","\$\$0","-1") & 10 \\
\hline if S.A("CHECKOVERFLOW,'EL ,EU,OVF) then & 11 \\
\hline S.A("P","\$\$0")="OVERFLOW" & 12 \\
\hline S.A("F","EXITSTATUS")="\#ERROR" & 13 \\
\hline exit sub & 14 \\
\hline end if & 15 \\
\hline ENDP=S.A("SetMark",EL,EU,EM,EF,"\$3","\$4","',"\$\$0","-1") & 16 \\
\hline if BEGP AND ENDP then & 17 \\
\hline if S.A("PL","\$1") < S.A("PL","\$3") then & 18 \\
\hline if len(MS) $>0$ and MS $<>$ "\#EMPTY" then & \\
\hline S.A("P","\$\$0")=MS & 19 \\
\hline ZZ=S.A("PL","\$4") -S.A("PL","\$2") & 20 \\
\hline if $Z Z<0$ then $Z Z=0$ & 21 \\
\hline S.A("P","\$0")=ZZ & 22 \\
\hline S.A("F","EXITSTATUS")="\#TRUE" & 23 \\
\hline else & 24 \\
\hline S.A("P","\$\$0")="MX_bf_MN" & 25 \\
\hline S.A("P","\$0")="0" & 26 \\
\hline S.A("F","EXITSTATUS")="\#FALSE" & 27 \\
\hline end if & 28 \\
\hline elseif BEGP OR ENDP then 'only one found & 29 \\
\hline S.A("P","\$\$0")="onlMNoMX" & 30 \\
\hline S.A("F","EXITSTATUS")="\#FALSE" & 31 \\
\hline S.A("P","\$0") = "0" & 32 \\
\hline else 'rising or descending & 33 \\
\hline S.A("P","\$0")= "0" & 34 \\
\hline S.A("F","EXITSTATUS")="\#FALSE" & 35 \\
\hline end if & 36 \\
\hline END SUB & 37 \\
\hline
\end{tabular}

(Manuscript received October 14, 2004;

revision accepted for publication March 29, 2005.) 